\title{
Intrauterine exposure and pharmacology of conventional thiopurine therapy in pregnant patients with inflammatory bowel disease
}

\author{
Bindia Jharap, ${ }^{1}$ Nanne K H de Boer, ${ }^{1}$ Pieter Stokkers, ${ }^{2,3}$ Daniel W Hommes, ${ }^{4,5}$ \\ Bas Oldenburg, ${ }^{6}$ Gerard Dijkstra, ${ }^{7}$ C Janneke van der Woude, ${ }^{8}$ Dirk J de Jong, ${ }^{9}$ \\ Chris J J Mulder, ${ }^{1}$ Ruurd M van Elburg, ${ }^{10}$ Adriaan A van Bodegraven, ${ }^{1}$ for the Dutch \\ Initiative on Crohn and Colitis
}

For numbered affiliations see end of article.

\section{Correspondence to}

Bindia Jharap, Department of Gastroenterology and Hepatology, VU University Medical Center, PO Box 7057, Amsterdam $1007 \mathrm{MB}$

The Netherlands;

b.jharap@vumc.nl

Received 28 August 2012 Revised 4 January 2013 Accepted 28 January 2013

Published Online First

19 February 2013
To cite: Jharap $B$, de Boer NKH, Stokkers $P$, et al. Gut 2014;63:451-457.

\section{ABSTRACT}

Objective Several studies have demonstrated a favourable safety profile for thiopurine use for inflammatory bowel disease (IBD) during pregnancy. We performed a study in pregnant patients with IBD who were using thiopurines, in order to determine the influence of pregnancy on thiopurine metabolism and to assess intrauterine exposure of the fetus to thiopurines.

Design Female patients with IBD receiving steady-state thiopurines and planning a pregnancy were prospectively enrolled. 6-Thioguanine nucleotide (6-TGN) and 6methylmercaptopurine (6-MMP) concentrations were determined, combined with routine laboratory tests, before, during and after pregnancy. Thiopurine metabolites were measured in umbilical cord blood immediately after delivery.

Results Thirty patients who were using azathioprine (28 patients, median dose $1.93 \mathrm{mg} / \mathrm{kg}$ ) or mercaptopurine (two patients, doses 1.32 and $0.94 \mathrm{mg} / \mathrm{kg}$ ) were included. During pregnancy, median 6-TGN decreased over time ( $p=0.001$ ). while 6-MMP increased, without causing myelotoxicity or hepatotoxicity. After delivery, both 6-TGN and 6-MMP levels returned to preconception baseline levels. Fetal 6-TGN concentrations correlated positively with maternal 6-TGN levels $(p<0.0001)$. No 6-MMP was detected in the newborns, except one born with pancytopenia and high alkaline phosphatase activity; the mother of this infant had severe pre-eclampsia. All infants had normal Apgar scores, but 60\% had anaemia at birth. No major congenital abnormalities were observed.

Conclusions Pregnancy has a major effect on maternal thiopurine metabolism. In utero the unborn child is exposed to 6-TGN, but not to 6-MMP. Sixty per cent of the infants were born with anaemia, which raises the question whether infants should be tested for possible anaemia immediately after birth.

\section{INTRODUCTION}

Conventional immune-modulating thiopurines such as mercaptopurine (MP) and its pro-drug, azathioprine (AZA), have proven efficacy in the treatment of inflammatory bowel disease (IBD), both inducing and, especially, maintaining clinical remission. ${ }^{1-3}$ Patients with IBD treated with thiopurines are often in their reproductive years, as IBD predominantly occurs in the second to third decade of life. ${ }^{4} 5$ Although animal studies have

\section{Significance of this study}

What is already known about this subject?

- Thiopurine use during pregnancy is relatively safe.

- The placenta forms a barrier to azathioprine and its metabolites.

What are the new findings?

- Pregnancy has a major effect on thiopurine metabolism without development of myelotoxicity or hepatotoxicity.

- The fetus is exposed to the pharmacologically active 6-thioguanine nucleotides, but not to 6-methylmercaptopurine.

- Sixty per cent of infants are born with mild anaemia, probably due to maternal thiopurine use.

How might it impact on clinical practice in the foreseeable future?

- Monitoring maternal thiopurine metabolite concentration during pregnancy may allow avoidance of high fetal 6-thioguanine nucleotide levels.

- Infants exposed to maternal thiopurine use during pregnancy should be routinely examined for signs of anaemia immediately after birth.

suggested that thiopurines may be teratogenic, several human studies have shown a good safety profile for thiopurine use during pregnancy in patients with IBD. ${ }^{6-10}$ However, the safety profile has to be balanced against the potential risks of disease relapse for mother and fetus if thiopurines are withdrawn, since a flare of IBD is associated with an increased risk of lower birth weight or preterm birth. ${ }^{11} 12$

In a small pilot study, our research group demonstrated in vivo that the fetus is exposed to the pharmacologically active 6-thioguanine nucleotides (6-TGN) generated by maternal AZA use. Interestingly, no 6-methylmercaptopurine (6-MMP) was detected in red blood cells (RBCs) of the three infants studied. ${ }^{13}$ 
Prospective data on pharmacokinetic effects of pregnancy on thiopurine metabolism are lacking. Therefore, the aims of this study of female patients with IBD were to examine the influence of pregnancy on thiopurine metabolism and determine intrauterine exposure to thiopurine metabolites and its potentially toxic effect on pregnancy outcome.

\section{MATERIALS AND METHODS}

\section{Patient population and baseline characteristics}

Female patients with IBD aged 18-45 years, who were receiving steady-state AZA or MP for at least 8 consecutive weeks and planning a pregnancy, were eligible for this prospective, Dutch multicentre study. Patients were included between January 2006 and January 2011. The study was approved by the medical ethics committee of all participating hospitals. All patients gave informed consent. Exclusion criteria were: concomitant use of allopurinol, ACE inhibitors or furosemide; bone marrow suppression (leucocyte and/or platelet count lower than the lower reference limit); presence of active inflammation (C-reactive protein $>1 \times$ upper normal limit in combination with fever); anaemia (haemoglobin concentration lower than the lower reference limit); impaired renal function (serum creatinine $>2 \times$ upper normal limit); elevated liver function tests $(>2 \times$ upper normal limit); known cardiac failure (New York Heart Association scale, grade III-IV); a blood transfusion received within the last 3 months; requested/planned delivery at home.

At baseline, the following data on patient characteristics were collected: weight, length, smoking, alcohol use, type of IBD (Crohn's disease (CD), ulcerative colitis (UC) or unclassified), duration of IBD at baseline, IBD localisation, nature of IBD, type and dose of thiopurine, and co-medication. All patients with UC or CD were classified according to the Montreal classification. ${ }^{14}$

\section{Thiopurine metabolite levels and toxicity of thiopurines during pregnancy}

6-TGN and 6-MMP were measured in RBCs by the validated method of Shipkova et al, ${ }^{15}$ which is a modification of the method of Dervieux et al. ${ }^{16}$ The blood samples obtained in lithium tubes sprayed with heparin were sent to the pharmacy department of the VU University Medical Center for determination of thiopurine metabolites. To minimise the risk of thiopurine metabolite concentrations decreasing because of their limited chemical stability, all samples were immediately stored at $4^{\circ} \mathrm{C}$ until shipment. ${ }^{17}$ Upon arrival at the pharmacy department, $\mathrm{RBCs}$ were washed and stored at $-20^{\circ} \mathrm{C}$ until analysis. In each sample, RBCs were isolated from the $7 \mathrm{ml}$ of drawn blood, yielding an RBC count of $\sim 3.5 \times 10^{12} / 1$ (range $2.5-4.5 \times 10^{12} / \mathrm{l}$ ). In the $250 \mu \mathrm{l}$ used for analysis, there were on average $8.8 \times 10^{8}$ RBCs. Metabolite concentrations were subsequently expressed in $\mathrm{pmol} / 8 \times 10^{8}$ RBCs. A reversed-phase gradient highperformance liquid chromatography system was used to separate 6-TGN and 6-MMP. ${ }^{17}$ The lower limit of detection of 6-TGN and 6-MMP was 30 and $100 \mathrm{pmol} / 8 \times 10^{8} \mathrm{RBCs}$, respectively.

To compare our data with previously published studies of patients with IBD, in which the method developed by Lennard and Singleton ${ }^{18}$ was usually used to determine thiopurine metabolites, 6-TGN concentrations were divided by 2.6. ${ }^{15}$ By means of this conversion, the concentrations derived with the method of Dervieux et al can be compared with Lennard-based levels (correlation coefficient $r=0.99) .{ }^{15}$ Throughout this article, these converted metabolite levels (and thus comparable with data generated by the method of Lennard and Singleton) will be primarily used. The outcomes according to both methods will be tabulated.
Thiopurine metabolite levels were measured at baseline (before pregnancy), directly after confirmation of pregnancy, at the end of the first and second trimesters, at delivery, and 3 months after delivery. Thiopurine dose was not changed throughout the study period. During these six planned visits, blood was routinely drawn to assess potential thiopurine-induced biochemical toxicity (eg, myelotoxicity, hepatotoxicity). Hepatotoxicity in the mothers was defined as twice the upper limit of alanine transaminase (ALT), aspartate transaminase (AST), alkaline phosphatase (AP) or $\gamma$-glutamyltransferase $(\gamma$-GT) activity or more. Myelotoxicity in the mothers was defined as the presence of leucopenia, thrombocytopenia or a haemoglobin level below the lower reference limit according to the WHO.

\section{Intrauterine exposure to thiopurine metabolites and toxicity in infants}

To determine intrauterine exposure to thiopurine therapy, blood was drawn from the artery and vein of the umbilical cord immediately after delivery. 6-TGN and 6-MMP levels were determined in these samples. ${ }^{17}$ In addition, blood was routinely drawn from infants to assess potential toxicity (hepatotoxicity and bone marrow depression) due to maternal use of thiopurines. In newborns, leucopenia was defined as a leucocyte count below $5 \times 10^{9} / 1$, anaemia as a haemoglobin level below $10 \mathrm{mmol} / \mathrm{l}$, and thrombocytopenia as a thrombocyte count below $150 \times 10^{9} / 1 .{ }^{19}$ Hepatotoxicity in newborns was defined as: $\mathrm{ALT}>50 \mathrm{U} / 1, \mathrm{AST}>140 \mathrm{U} / \mathrm{l}, \mathrm{AP}>300 \mathrm{U} / 1$ or $\gamma-\mathrm{GT}>263 \mathrm{U} / \mathrm{l}^{20}$

\section{PREGNANCY OUTCOME}

Data on pregnancy outcome included gestational age, premature births (gestational age $<37$ weeks), birth weight, caesarean section, congenital malformations and spontaneous abortion.

\section{Statistical analysis}

Demographic data are given descriptively and are tabulated. Data are expressed as median (IQR) according to distribution. The Spearman rank correlation coefficient was used to assess correlations. The Friedman test was used to assess statistically significant differences in thiopurine metabolite concentration over time during pregnancy. Post hoc analysis was conducted with the Wilcoxon signed-rank test for paired data. A p value less than 0.05 was considered significant. SPSS Version 17.0 was used for statistical analysis. ${ }^{21}$

\section{RESULTS}

\section{Patient population and baseline characteristics}

In total, 59 female patients with IBD were included on the basis of their expressed wish to become pregnant. Of these patients, $41(69 \%)$ became pregnant. Seven patients had a miscarriage in the first trimester, while another three had a spontaneous abortion in the second trimester. One patient was lost to follow-up. In total, 30 patients had a successful pregnancy outcome and were further analysed for the purpose of this study. Patient characteristics of these 30 patients are given in table 1 . Median age of the patients was 30 years (IQR 27-33). CD and UC were diagnosed in $24(80 \%)$ and six (20\%) patients, respectively. Median duration of IBD at the time of inclusion was 8.5 years (IQR 6.0-12.0).

Characteristics of thiopurine therapy are also provided in table 1. Twenty-eight patients were receiving steady-state AZA at a median dose of $1.93 \mathrm{mg} / \mathrm{kg}$ (IQR 1.46-2.23). Two patients were receiving steady-state MP at a dose of 1.32 and $0.94 \mathrm{mg} / \mathrm{kg}$, respectively. 
Table 1 Patient characteristics (mothers) (total=30)

\begin{tabular}{ll}
\hline Characteristic & Value \\
\hline Age (years) & $30(27-33)$ \\
Weight (kg) & $70(57-78)$ \\
Smoking at inclusion & 5 \\
Alcohol use at inclusion & 9 \\
Disease (CD/UC) & $24 / 6$ \\
Montreal classification of UC patients ( $\mathrm{n}=6$ ) & \\
E2 & 3 \\
E3 & 3 \\
Montreal classification of CD patients ( $\mathrm{n}=24)$ & \\
A1/A2/A3 & $7 / 17 / 0$ \\
L1/L2/L3 & $7 / 7 / 10$ \\
B1/B2/B1 $/$ B2p/missing & $11 / 4 / 7 / 1 / 1$ \\
Median duration of IBD (years) & $8.5(6.0-12.0)$ \\
Type of thiopurine & \\
AZA & 28 \\
MP & 2 \\
Dose of AZA (mg/kg) & $1.93(1.46-2.23)$ \\
Dose of MP (mg/kg) & \\
Patient 1 & 1.32 \\
Patient 2 & 0.94 \\
Co-medication at inclusion & \\
Mesalazine & 6 \\
Prednisone enema & 2 \\
Infliximab & 2 \\
Adalimumab & 1 \\
Laxatives & 2 \\
\hline Values are number or median (IQR). \\
AZA, azathioprine; CD, Crohn's disease; IBD, inflammatory bowel disease; MP, \\
mercaptopurine; UC, ulcerative colitis. \\
\end{tabular}

\section{Thiopurine levels and toxicity during pregnancy}

Overall, a statistically significant decrease in median 6-TGN levels was observed during pregnancy $(p=0.001)$. After delivery, median 6-TGN levels returned to preconception baseline levels (figure 1 and table 2). In contrast, median 6-MMP levels increased during pregnancy, but the increase was not significant $(p=0.183)$. 6-MMP levels had returned to preconception baseline concentrations 3 months after delivery (figure 2 and table 2). In $10 \%$ of the women, only a slight alteration or no change in 6-MMP or 6-TGN levels was observed during pregnancy. Median 6-MMP/6-TGN ratios increased significantly during pregnancy $(p=0.007)$. After delivery, the ratio decreased to preconception baseline levels (figure 3 and table 2). The significant differences in thiopurine metabolite concentration during pregnancy and post partum compared with preconception values are shown in table 3. In none of the 30 patients were biochemical signs of myelotoxicity or hepatotoxicity observed during pregnancy. All patients tolerated thiopurine therapy well and no adverse events were reported.

There was no clear difference in recalculated 6-TGN levels between patients with a full-term pregnancy and patients who had a miscarriage in the first trimester $\left(106 \mathrm{vs} 96 \mathrm{pmol} / 8 \times 10^{8}\right.$ $\mathrm{RBCs}$, respectively) or spontaneous abortion in the second trimester ( 85 vs $96 \mathrm{pmol} / 8 \times 10^{8} \mathrm{RBCs}$, respectively). However, there was a difference in 6-MMP levels between patients with a full-term pregnancy and patients who had a miscarriage in the first trimester ( 2140 vs $340 \mathrm{pmol} / 8 \times 10^{8} \mathrm{RBCs}$, respectively). The 6-MMP levels in the second trimester in women with a fullterm pregnancy and those who had a spontaneous abortion

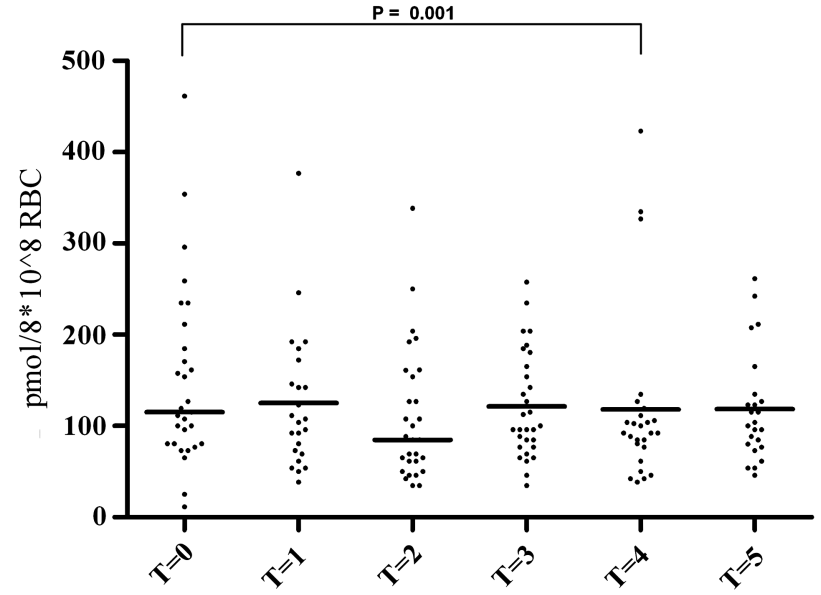

Figure 1 Maternal 6-thioguanine nucleotide (6-TGN) levels. A significant decrease in median maternal 6-TGN (indicated by horizontal lines) was observed during pregnancy $(p=0.001)$. After delivery, 6-TGN levels returned to baseline concentrations (Wilcoxon, $T=0$ vs $T=5$, $\mathrm{p}>0.05$ ). 6 -TGN concentration ( $\mathrm{pmol} / 8 \times 10^{8}$ red blood cells $(\mathrm{RBC})$ ) is depicted on the $y$-axis. $T=0$, before conception; $T=1$, directly after pregnancy confirmation; $\mathrm{T}=2$, after the first trimester; $\mathrm{T}=3$, after the second trimester; $T=4$, delivery; $T=5,3$ months after delivery. 6-TGN concentrations are expressed in converted values (comparable with data generated according to the method of Lennard).

were equivalent ( 2330 vs $2830 \mathrm{pmol} / 8 \times 10^{8} \mathrm{RBCs}$, respectively). No further statistical tests were performed, as the groups of patients were too small for proper analysis.

\section{Thiopurine levels and blood results in infants}

Thirty women gave birth to 31 infants, as one pregnancy resulted in twins. Because of technical or logistic problems, umbilical cord blood was not drawn from six infants. Therefore, thiopurine metabolites were determined in $25 / 31$ infants (81\%). Median 6-TGN concentration was lower in the newborn infants than in mother at the time of delivery: 42 vs $92 \mathrm{pmol} / 8 \times 10^{8} \mathrm{RBCs}, \mathrm{p}=0.01$ (equivalent to the originally measured 100 vs $240 \mathrm{pmol} / 8 \times 10^{8} \mathrm{RBCs}$, according to the method of Dervieux et al). 6-TGN levels in the infants correlated strongly and positively with 6-TGN levels in the mother (Spearman correlation $\mathrm{r}=0.74, \mathrm{p}<0.0001$ ) (figure 4). No 6-MMP was detected in 24 of the 25 infants (96\%). The remaining infant had a 6-MMP concentration of $1290 \mathrm{pmol} / 8 \times 10^{8} \mathrm{RBCs}$ and a 6 -TGN level of $65 \mathrm{pmol} / 8 \times 10^{8}$ RBCs; this child was born with pancytopenia and a 10 -fold increased AP activity at a gestational age of 38 weeks. The mother of this infant had a 6-MMP concentration of $2200 \mathrm{pmol} / 8 \times 10^{8} \mathrm{RBCs}$ during delivery. She received infliximab combined with AZA until 29 weeks of gestation and developed severe pregnancy pre-eclampsia, leading to early hospital admittance. Haemoglobin level was measured in 16 of the 31 $(52 \%)$ newborns. Ten of these $(63 \%)$ had anaemia at birth (median haemoglobin level $9.25 \mathrm{mmol} / \mathrm{l}$ (IQR 8.25-9.60)). Two of these children had combined anaemia and thrombocytopenia. Median 6-TGN concentration was higher in infants with anaemia than in those without: 100 vs $35 \mathrm{pmol} / 8 \times 10^{8} \mathrm{RBCs}$, respectively, $\mathrm{p}=0.22$ ( $260 \mathrm{vs} 90 \mathrm{pmol} / 8 \times 10^{8} \mathrm{RBCs}$, respectively, according to the method of Dervieux et al). All newborns with anaemia had normal Apgar scores immediately after birth. AP activity was measured in 12 of the 31 newborns. Three of these (25\%) had an isolated AP activity above the reference limit; 
Table 2 Pharmacokinetics of thiopurine use during pregnancy

\begin{tabular}{|c|c|c|c|c|}
\hline & 6-TGN (Lennard method) & 6-TGN (Dervieux method) & 6-MMP & 6-MMP/6-TGN ratio (Lennard method) \\
\hline Pre-conception & 115 (81-198) & $280(210-550)$ & $1290(584-2790)$ & $10.9(4.2-24.4)$ \\
\hline After pregnancy confirmation & $106(70-166)$ & $270(190-380)$ & $2140(820-4548)$ & $22.6(9.1-37.4)$ \\
\hline Gestational week 13.5 & 85 (56-158) & $220(130-500)$ & $2330(615-4390)$ & $27.9(4.8-48.5)$ \\
\hline Gestational week 26.5 & $98(77-169)$ & $230(170-260)$ & $2648(468-5888)$ & $27.1(3.1-78.1)$ \\
\hline Delivery & $92(73-113)$ & $240(210-290)$ & $2390(268-6588)$ & $20.3(1.3-90.1)$ \\
\hline 3 months after delivery & $102(78-133)$ & $270(190-550)$ & $1090(518-3590)$ & $13.8(5.1-27.5)$ \\
\hline
\end{tabular}

however, all three infants were born prematurely. No 6-MMP was detected in these three children. There was no significant difference in 6-TGN concentrations in blood obtained from the umbilical vein compared with the umbilical artery.

\section{Mode of delivery, infant characteristics and congenital abnormalities}

A caesarean section was performed in 14 of the $30(47 \%)$ mothers. Ten $(71 \%)$ had an elective caesarean section. Reasons were perianal disease, history of abdominal or vaginal surgery, previous caesarean section, and pre-eclampsia. All emergency caesarean sections $(n=4)$ were performed because of fetal distress. Median gestational age was 39 weeks (IQR 38-40.1), and median birth weight was $3410 \mathrm{~g}$ (IQR 3200-3680). In total, two of the $30(7 \%)$ patients delivered prematurely; one of them had a twin pregnancy. The preterm twins were born at a gestational age of 33 weeks with birth weights of 1874 and $1809 \mathrm{~g}$, while one infant was born at a gestational age of 31 weeks with a birth weight of $2030 \mathrm{~g}$. Two newborns (6.5\%) had congenital abnormalities, which consisted of a clubfoot and an isolated ptosis of the left eye. The converted 6-TGN concentrations in these infants were 77 and $42 \mathrm{pmol} / 8 \times 10^{8} \mathrm{RBCs}$, respectively.

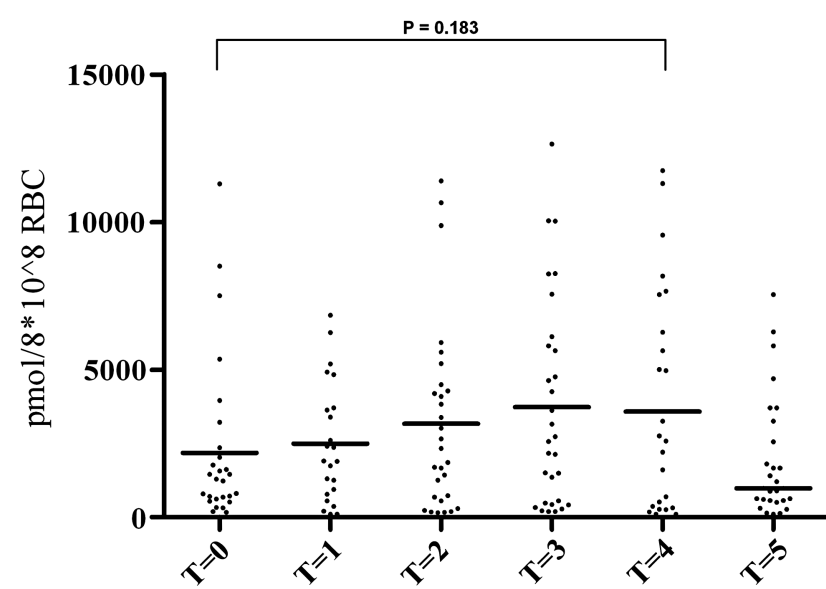

Figure 2 Maternal 6-methylmercaptopurine (6-MMP) levels. An increase in median maternal 6-MMP levels (indicated by horizontal lines) was observed during pregnancy, which was not significant $(p=0.183)$. After delivery, 6-MMP levels returned to baseline concentrations (Wilcoxon, $\mathrm{T}=0$ vs $\mathrm{T}=5, \mathrm{p}>0.05$ ). 6-MMP concentration (pmol/8 $8 \times 10^{8}$ red blood cells (RBC)) is depicted on the $y$-axis. $T=0$, before conception; $T=1$, directly after pregnancy confirmation; $T=2$, after the first trimester; $\mathrm{T}=3$, after the second trimester; $\mathrm{T}=4$, delivery; $\mathrm{T}=5,3$ months after delivery.

\section{DISCUSSION}

Our study demonstrates that pregnancy has an important effect on maternal thiopurine metabolism, leading to decreased 6-TGN and increased 6-MMP concentrations during stable dosing of thiopurines. The fetus is exposed to 6-TGN, with concentrations that correlate strongly with maternal 6-TGN concentrations. Remarkably, in only one infant, who was diagnosed with myelosupression and liver function test abnormalities at birth, was 6-MMP detected. Mild anaemia was observed directly after birth in $60 \%$ of the newborns.

During pregnancy, a decrease in median 6-TGN concentration was observed, while 6-MMP levels increased. This alteration in thiopurine metabolism did not lead to biochemical toxicity or adverse events in the mothers. After delivery, both 6-TGN and 6-MMP levels returned to baseline. Previous studies have shown that hormonal changes during pregnancy may influence drugmetabolising enzymes. ${ }^{22}$ An increase in thiopurine S-methyl transferase (TPMT) activity during pregnancy, causing preferential metabolism of MP to 6-MMP at the expense of 6-TGN formation, may explain the decrease in 6-TGN and increase in

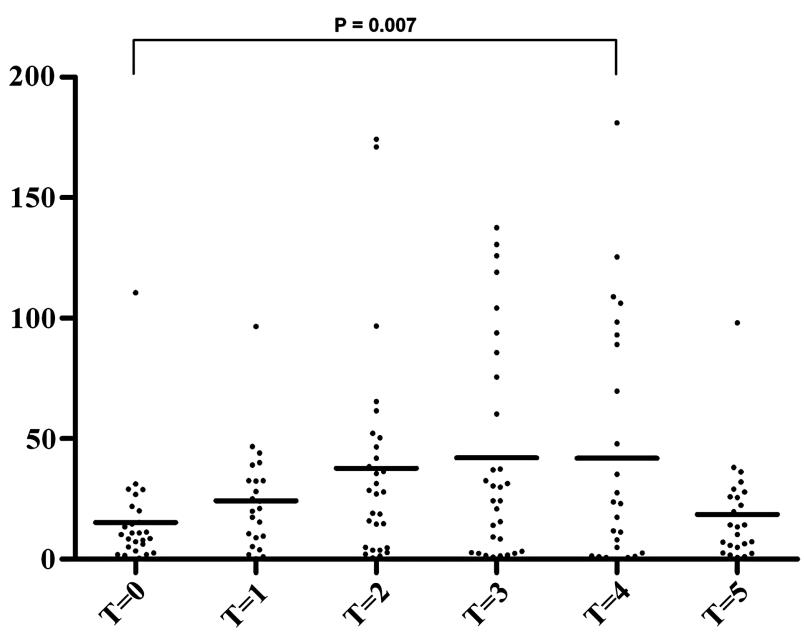

Figure 3 Maternal 6-methylmercaptopurine (6-MMP)/6-thioguanine nucleotide (6-TGN) ratios. A significant increase in median maternal 6-MMP/6-TGN ratio (indicated by horizontal lines) was observed during pregnancy $(p=0.007)$. After delivery, it returned to baseline levels (Wilcoxon, $T=0$ vs $T=5, p>0.05$ ). 6-MMP/6-TGN ratio is depicted on the $\mathrm{y}$-axis. $\mathrm{T}=0$, before conception; $\mathrm{T}=1$, directly after pregnancy confirmation; $\mathrm{T}=2$, after the first trimester; $\mathrm{T}=3$, after the second trimester; $\mathrm{T}=4$, delivery; $\mathrm{T}=5,3$ months after delivery. The 6 -TGN concentrations are expressed in converted values (comparable with data generated according to the method of Lennard). 
Table 3 Change in thiopurine metabolite levels before, during and after pregnancy

\begin{tabular}{lccl}
\hline Time & $\Delta$ 6-TGN† (\%) & $\Delta 6-M M P(\%)$ & $\Delta 6-M M P / 6-T G N+$ ratio $(\%)$ \\
\hline $\mathrm{T}=0$ and $\mathrm{T}=1$ & $-9(8 \%)$ & $+850(66 \%)$ & $+12(107 \%)$ \\
$\mathrm{T}=0$ and $\mathrm{T}=2$ & $-30(26 \%)^{*}$ & $+1040(81 \%)$ & $+17(156 \%)^{*}$ \\
$\mathrm{~T}=0$ and $\mathrm{T}=3$ & $-17(15 \%)$ & $+1358(105 \%)$ & $+16(149 \%)^{*}$ \\
$\mathrm{~T}=0$ and $\mathrm{T}=4$ & $-23(20 \%)$ & $+1100(85 \%)$ & $+9(86 \%)$ \\
$\mathrm{T}=0$ and $\mathrm{T}=5$ & $-13(11 \%)$ & $-200(16 \%)$ & $+3(27 \%)$
\end{tabular}

Post hoc analysis of thiopurine metabolite concentration during pregnancy and post partum compared with prepregnancy values, expressed as an absolute number (in pmol $/ 8 \times 10^{8}$ red blood cells) and percentage. $T=0$, before conception; $T=1$, directly after pregnancy confirmation; $\mathrm{T}=2$, after the first trimester; $\mathrm{T}=3$, after the second trimester; $\mathrm{T}=4$, delivery; $\mathrm{T}=5,3$ months after delivery.

${ }^{*} \mathrm{p}<0.05$.

+Converted 6-TGN levels are used.

6-MMP, 6-methylmercaptopurine; 6-TGN, 6-thioguanine nucleotide.

6-MMP. As thiopurine metabolite levels are the essential pharmacological end products of the complex thiopurine metabolism, these levels were measured during pregnancy rather than possible changes in TPMT or other thiopurine-metabolising enzyme activities. Of note, as all the ratios of the metabolite concentrations of 6-MMP and 6-TGN at baseline were within 4-11 and all mothers tolerated the drug well before inclusion in the study, we virtually excluded abnormally low TPMT activity due to mutant TPMT alleles (genotype) in our cohort. However, no definite conclusions can be drawn on the basis of our study design about how exactly thiopurine metabolism is altered during pregnancy.

The risk of developing biochemical signs of hepatotoxicity has been shown to increase when 6-MMP levels exceed $5700 \mathrm{pmol} / 8 \times 10^{8} \mathrm{RBCs}$ in non-pregnant patients with IBD. ${ }^{24}$ A 6-MMP level above $5700 \mathrm{pmol} / 8 \times 10^{8} \mathrm{RBCs}$ was measured in $26 \%$ of our patients during pregnancy, but none developed liver function test abnormalities. It has been suggested that the 6-MMP/6-TGN ratio may be a predictor of therapeutic efficacy, as a ratio above 11 has been associated with therapeutic

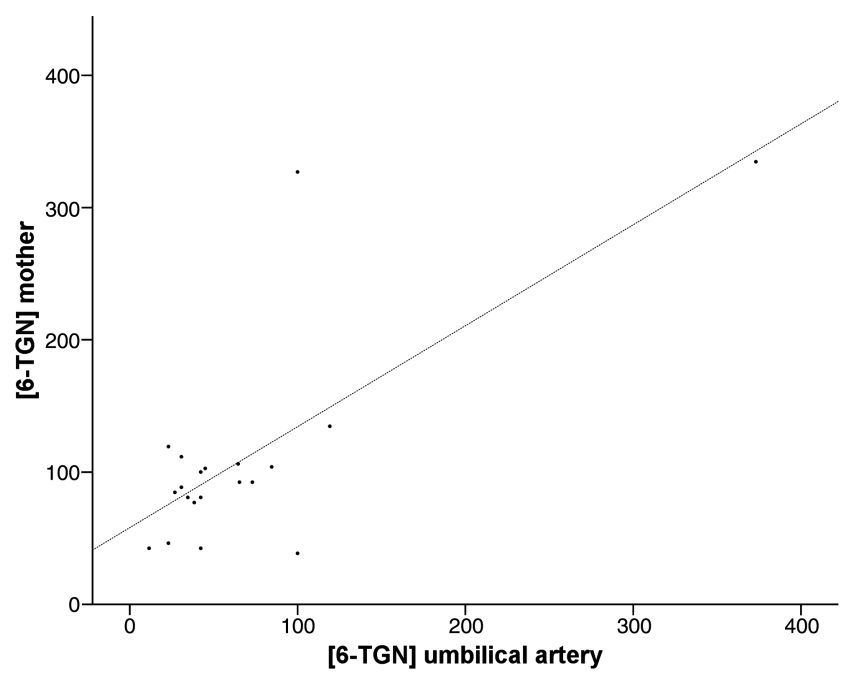

Figure 4 Correlation between 6-thioguanine nucleotide (6-TGN) levels in mother and child. There was a strong correlation $(p<0.0001)$

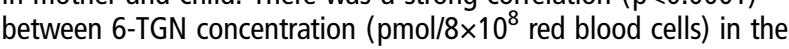
mother at the time of delivery (y-axis) and in the infant (x-axis). 6-TGN concentrations are expressed in converted values (comparable with data generated by the method of Lennard). inefficacy. ${ }^{24}$ Although the median 6-MMP/6-TGN ratio was above 11 during pregnancy, none of the women in our series had a clinical relapse of IBD.

Our study confirms that the fetus is exposed to 6-TGN during maternal use of thiopurines during pregnancy. Data on the transfer of thiopurines or its metabolites across the placenta are limited. Saarikoski and Seppala ${ }^{25}$ demonstrated that the parent drug, AZA, and its inactive metabolite, thiouric acid, can pass through the human placenta, as both compounds were found in fetal blood after administration of radioactively labelled AZA to three pregnant women. Interestingly, AZA and MP concentrations in the fetal blood were $1-5 \%$ and $1-2 \%$, respectively, compared with maternal blood levels, which suggests that the placenta serves as a barrier to thiopurines and its metabolites. ${ }^{25}$ In line with this, Hutson and colleagues ${ }^{26}$ showed, in a dually perfused experimental placenta model, that maternal pharmacokinetics and placental binding limit the transfer of MP from mother to child. They also demonstrated that the placenta has limited capacity to convert MP into pharmacologically active metabolites, which suggests that the maternally derived metabolites cross the placental barrier and not the parent drug. In our study, there was no difference in 6-TGN concentration in blood from the umbilical vein compared with that from the umbilical artery, which may indicate that no additional thiopurine metabolism takes place in the fetus. $^{27}{ }^{28}$ However, we need to be cautious in drawing any conclusions about this on the basis of our study design and outcomes. As fetal 6-TGN levels correlated strongly with maternal 6-TGN concentrations, therapeutic drug monitoring in pregnant women should perhaps be considered to avoid extremely elevated maternal and fetal 6-TGN levels and thus potential thiopurine toxicity.

No 6-MMP levels were detected in 24 of the 25 infants. Remarkably, one child had a 6-MMP level of $1290 \mathrm{pmol} / 8 \times 10^{8}$ RBCs, allegedly inducing pancytopenia and a 10 -fold increase in AP activity. His mother had severe pre-eclampsia during pregnancy, which resulted in hospital admittance and an emergency caesarian section at 38 weeks of gestation. Pre-eclampsia may cause erythrocythaemia and severe thrombocytopenia in newborns or fetal hyperviscosity-polycythaemia syndrome, all associated with increased placental leakage and impaired function. $^{29} 30$ The pre-eclampsia in our patient may have resulted in increased permeability or disturbed semipermeability of the placental barrier to 6-MMP. ${ }^{31}$ Therefore, although speculative, the myelotoxicity in the above described newborn may be explained by the inhibition of de novo purine synthesis, caused by the presence of methylated MP derivatives (6-MMP). ${ }^{32} 33$

Sixty per cent of all the infants exposed to maternal thiopurine use during pregnancy had anaemia at birth. There are many possible reasons for this, considering that the mothers had IBD and often took concomitant medication, and many other potential causes. However, the common denominator, maternal thiopurine use, and the demonstrated intrauterine exposure to 6-TGN (and potentially other thiopurine metabolites) are the most obvious explanations. Therefore, we recommend that all children of thiopurine-using mothers should be tested for possible anaemia after birth.

In this study, median gestational age and birth weight were within normal ranges compared with the data from the 10-year perinatal prospective registration in the Netherlands. ${ }^{34}$ Two women $(7 \%)$ delivered prematurely, which is a lower rate than the $21.8 \%$ reported in a recent French collaborative study that evaluated pregnancy outcome of women with IBD exposed to thiopurines. ${ }^{7}$ The prevalence of congenital abnormalities of 
$6.5 \%$ in our study is higher than that of the average Dutch population and the reported $3.6 \%$ in the thiopurine-using IBD cohort from France. $^{7}$ However, the data on pregnancy outcome in our study are limited by the small sample of patients with IBD included, which may explain the conflicting data with regard to other larger studies. Considering the relatively high rate of miscarriage and spontaneous abortions $(24 \%)$ in our series, which are in line with previously reported data, further investigation of risk factors seems warranted. ${ }^{7}$ Importantly, the observed (high) percentage of miscarriage and spontaneous abortions in this study could not be related to (toxic) levels of thiopurine metabolites.

In conclusion, this study demonstrates that pregnancy has a major effect on thiopurine metabolism, leading to a decrease in 6-TGN and an increase in 6-MMP levels without causing myelotoxicity or hepatotoxicity. The fetus is exposed in utero to 6-TGN during maternal thiopurine use. 6-MMP was detected in only one child, probably because of placental insufficiency, whereas all other children had undetectable concentrations of 6-MMP at birth. Although all infants were exposed to 6-TGN, no major teratogenicity was observed. Sixty per cent of the infants had anaemia, probably due to maternal thiopurine use. The strong correlation between fetal and maternal 6-TGN concentration suggests that potentially toxic fetal 6-TGN levels can be avoided by monitoring maternal thiopurine metabolite concentration during pregnancy. In addition, our results raise the question whether all infants exposed to maternal thiopurine use during pregnancy should be routinely examined for signs of anaemia immediately after birth.

\author{
Author affiliations \\ ${ }^{1}$ Department of Gastroenterology and Hepatology, VU University Medical Center, \\ Amsterdam, The Netherlands \\ 2Department of Gastroenterology and Hepatology, Academic Medical Center, \\ Amsterdam, The Netherlands \\ ${ }^{3}$ Sint Lucas Andreas Hospital, Amsterdam, The Netherlands \\ ${ }^{4}$ Department of Gastroenterology and Hepatology, Leiden University Medical Center, \\ Leiden, The Netherlands \\ ${ }^{5}$ UCLA Health System, Los Angeles, California, USA \\ ${ }^{6}$ Department of Gastroenterology and Hepatology, University Medical Center Utrecht, \\ Utrecht, The Netherlands \\ ${ }^{7}$ Department of Gastroenterology and Hepatology, University Medical Center \\ Groningen, University of Groningen, Groningen, The Netherlands \\ ${ }^{8}$ Department of Gastroenterology and Hepatology, Erasmus University Medical \\ Center, Rotterdam, The Netherlands \\ ${ }^{9}$ Department of Gastroenterology and Hepatology, Radboud University Medical \\ Center, Nijmegen, The Netherlands \\ ${ }^{10}$ Department of Pediatrics, VU University Medical Center, Amsterdam, \\ The Netherlands
}

Acknowledgements We thank Dirk van Asseldonk, Margien Seinen and Esme Clasquin for their valuable assistance during the study.

Contributors BJ, NKHdB and AAvB had full access to all data of the study and take responsibility for the integrity of the data and the accuracy of the data analysis. Study concept and design: BJ, NKHdB, AAvB. Acquisition of data: BJ, NKHdB, PS, DWH, BO, GD, CJvdW, DJdJ, AAvB. Analysis and interpretation of data: BJ, NKHdB, RMvE, AAvB. Drafting of the manuscript: BJ. Critical revision of the manuscript for important intellectual content: BJ, NKHdB, PS, DWH, BO, GD, CJvdW, DJdJ, CJJM, RMvE, AAvB. Statistical analysis: BJ. Obtaining funding (Gastrostart): BJ, NKHdB, AAvB. Administrative, technical, or material support: BJ, NKHdB, AAvB.

Funding The study was partly funded by a restricted grant (Gastrostart) from the Dutch Gastroenterology Association.

Competing interests None.

Patient consent Obtained.

Ethics approval Medical ethics board of VU University Medical Center and the medical ethics boards of all other included hospitals.

Provenance and peer review Not commissioned; externally peer reviewed.

\section{REFERENCES}

1 Present DH, Korelitz BI, Wisch N, et al. Treatment of Crohn's disease with 6-mercaptopurine. A long-term, randomized, double-blind study. N Engl J Med 1980;302:981-7.

2 Sandborn W, Sutherland L, Pearson D, et al. Azathioprine or 6-mercaptopurine for inducing remission of Crohn's disease. Cochrane Database Syst Rev 2000;(2): CD000545.

3 Timmer A, McDonald JW, Macdonald JK. Azathioprine and 6-mercaptopurine for maintenance of remission in ulcerative colitis. Cochrane Database Syst Rev 2007; (1):CD000478.

4 Shivananda S, Lennard-Jones J, Logan $\mathrm{R}$, et al. Incidence of inflammatory bowel disease across Europe: is there a difference between north and south? Results of the European Collaborative Study on Inflammatory Bowel Disease (EC-IBD). Gut 1996;39:690-7

5 Vind I, Riis L, Jess T, et al. Increasing incidences of inflammatory bowel disease and decreasing surgery rates in Copenhagen City and County, 2003-2005: a population-based study from the Danish Crohn colitis database. Am J Gastroenterol 2006;101:1274-82

6 Akbari M, Shah S, Velayos FS, et al. Systematic review and meta-analysis on the effects of thiopurines on birth outcomes from female and male patients with inflammatory bowel disease. Inflammatory Bowel Disease 2013;19:15-22.

7 Coelho J, Beaugerie L, Colombel JF, et al. Pregnancy outcome in patients with inflammatory bowel disease treated with thiopurines: cohort from the CESAME Study. Gut 2011;60:198-203.

8 Francella A, Dyan A, Bodian C, et al. The safety of 6-mercaptopurine for childbearing patients with inflammatory bowel disease: a retrospective cohort study. Gastroenterology 2003;124:9-17.

9 Polifka JE, Friedman JM. Teratogen update: azathioprine and 6-mercaptopurine. Teratology 2002;65:240-61.

10 Shim L, Eslick GD, Simring AA, et al. The effects of azathioprine on birth outcomes in women with inflammatory bowel disease (IBD). J Crohns Colitis 2011;5:234-8.

11 Bush MC, Patel S, Lapinski RH, et al. Perinatal outcomes in inflammatory bowel disease. J Matern Fetal Neonatal Med 2004;15:237-41.

12 Reddy D, Murphy SJ, Kane SV, et al. Relapses of inflammatory bowel disease during pregnancy: in-hospital management and birth outcomes. Am J Gastroenterol 2008;103:1203-9.

13 de Boer NK, Jarbandhan SV, de Graaf $\mathrm{P}$, et al. Azathioprine use during pregnancy: unexpected intrauterine exposure to metabolites. Am J Gastroenterol 2006;101:1390-2.

14 Satsangi J, Silverberg MS, Vermeire S, et al. The Montreal classification of inflammatory bowel disease: controversies, consensus, and implications. Gut 2006;55:749-53

15 Shipkova M, Armstrong VW, Wieland E, et al. Differences in nucleotide hydrolysis contribute to the differences between erythrocyte 6-thioguanine nucleotide concentrations determined by two widely used methods. Clin Chem 2003:49:260-8.

16 Dervieux T, Meyer G, Barham R, et al. Liquid chromatography-tandem mass spectrometry analysis of erythrocyte thiopurine nucleotides and effect of thiopurine methyltransferase gene variants on these metabolites in patients receiving azathioprine/6-mercaptopurine therapy. Clin Chem 2005;51:2074-84.

17 de Graaf P, Vos RM, de Boer NH, et al. Limited stability of thiopurine metabolites in blood samples: relevant in research and clinical practise. J Chromatogr B Analyt Technol Biomed Life Sci 2010;878:1437-42.

18 Lennard L, Singleton HJ. High-performance liquid chromatographic assay of the methyl and nucleotide metabolites of 6 -mercaptopurine: quantitation of red blood cell 6-thioguanine nucleotide, 6-thioinosinic acid and 6-methylmercaptopurine metabolites in a single sample. J Chromatogr 1992;583:83-90.

19 Pekelharing JM, Souverijn JHM, Hooijkaas H, et al. Handboek medische laboratoriumdiagnostiek. Houten, the Netherlands: Prelum publisher, 2009.

20 Rosenthal P. Assessing liver function and hyperbilirubinemia in the newborn. National Academy of Clinical Biochemistry. Clin Chem 1997;43:228-34.

21 Statistical Package for Social Sciences. Chicago, 2008.

22 Hodge LS, Tracy TS. Alterations in drug disposition during pregnancy: implications for drug therapy. Expert Opin Drug Metab Toxicol 2007;3:557-71.

23 Jeong $\mathrm{H}$. Altered drug metabolism during pregnancy: hormonal regulation of drug-metabolizing enzymes. Expert Opin Drug Metab Toxicol 2010;6:689-99.

24 Dubinsky MC, Yang H, Hassard PV, et al. 6-MP metabolite profiles provide a biochemical explanation for 6-MP resistance in patients with inflammatory bowel disease. Gastroenterology 2002;122:904-15.

25 Saarikoski S, Seppala M. Immunosuppression during pregnancy: transmission of azathioprine and its metabolites from the mother to the fetus. Am J Obstet Gynecol 1973;115:1100-16.

26 Hutson JR, Lubetsky A, Walfisch A, et al. The transfer of 6-mercaptopurine in the dually perfused human placenta. Reprod Toxicol 2011;32:349-53. 
27 McLeod HL, Krynetski EY, Wilimas JA, et al. Higher activity of polymorphic thiopurine S-methyltransferase in erythrocytes from neonates compared to adults. Pharmacogenetics 1995;5:281-6.

28 Vettenranta K, Raivio KO. Key enzymes of purine degradation and reutilization in human fetal liver and brain. Biol Neonate 1990:58:311-17.

29 Heilmann L, Rath W, Pollow K. Fetal hemorheology in normal pregnancy and severe preeclampsia. Clin Hemorheol Microcirc 2005;32:183-90.

30 Huang SC, Chang FM. The adverse effect on fetal hemogram by preeclampsia: marked anisocytosis with normocytic, normochromic erythrocythemia as well as thrombocytopenia. Early Hum Dev 1994;37:91-8.
31 Salafia CM, Pezzullo JC, Lopez-Zeno JA, et al. Placental pathologic features of preterm preeclampsia. Am J Obstet Gynecol 1995;173:1097-105.

32 Bokkerink JP, Stet EH, De Abreu RA, et al. 6-Mercaptopurine: cytotoxicity and biochemical pharmacology in human malignant T-lymphoblasts. Biochem Pharmacol 1993:45:1455-63.

33 Dervieux T, Blanco JG, Krynetski EY, et al. Differing contribution of thiopurine methyltransferase to mercaptopurine versus thioguanine effects in human leukemic cells. Cancer Res 2001;61:5810-16.

34 Foundation Perinatal Registration. Ten years of perinatal registration in the Netherlands. Utrecht: Foundation Perinatal Registration the Netherlands, 2011. 


\section{GUT Intrauterine exposure and pharmacology of conventional thiopurine therapy in pregnant patients with inflammatory bowel disease}

Bindia Jharap, Nanne K H de Boer, Pieter Stokkers, et al.

Gut 2014 63: 451-457 originally published online February 19, 2013 doi: 10.1136/gutjnl-2012-303615

Updated information and services can be found at:

http://gut.bmj.com/content/63/3/451.full.html

These include:

References This article cites 29 articles, 7 of which can be accessed free at: http://gut.bmj.com/content/63/3/451.full.html\#ref-list-1

Email alerting Receive free email alerts when new articles cite this article. Sign up in service the box at the top right corner of the online article.

Notes

To request permissions go to:

http://group.bmj.com/group/rights-licensing/permissions

To order reprints go to:

http://journals.bmj.com/cgi/reprintform

To subscribe to BMJ go to:

http://group.bmj.com/subscribe/ 